\title{
New application of Thermal Ionization Mass Spectrometry: Sr-90 determination in environmental samples
}

\author{
S.K.SAHOO, N. KÁVÁSI AND T.AONO
}

National Institutes for Quantum and Radiological Science and Technology, Japan

In recent years, owing to significant developments on sample introduction, detector and interference removal techniques, many successful Sr-90 measurments have been reported using mass spectrometry instruments. For this purpose, inductively coupled plasma mass spectrometry (ICPMS) instruments with collision/reaction cells or triple quadrupole system have been primarily applied. Alternatively, thermal ionisation mass spectrometry (TIMS) is a robust technique for $\mathrm{Sr}-90$ determination.

The main advantages of the mass spectrometry method over the radiometric are the shorter analysis time, higher sample throughput and less sample intake. The low level Sr-90 detection in environmental samples with mass spectrometry instrument is a challenging task. The first critical point is the isobaric interference of $\mathrm{Zr}-90$ as a consequence of significant amounts of $\mathrm{Zr}$ in the environment.

The second critical point for the application of mass spectrometry instruments for Sr-90 determination is the peak tailing on the higher mass side from $\mathrm{Sr}-88$.

Considering the need of rapid and precise $\mathrm{Sr}-90$ determination after the Fukushima nuclear accident, a Sr-90 analysis method was developed using the Phoenix X62 TIMS in the laboratory of the National Institutes for Quantum and Radiological Science and Technology (QST), Japan. This is the first mass spectrometry method which was evaluated and confirmed by an independent proficiency test.

In this work, efforts were made to decrease the sample intake of stable strontium from $\mu \mathrm{g}$ to $\mathrm{ng}$ level, which can give the possibility of strontium isotope ratio analysis in low sample amount (mg level). This result can be interesting for meteorite as well as rock and sediment analysis. 